\title{
Hyperglycaemia in critically ill patients: the immune system's sweet tooth
}

\author{
Gustav van Niekerk ${ }^{*}$ D, Tanja Davis and Anna-Mart Engelbrecht
}

\begin{abstract}
There is an ongoing debate regarding the efficacy of glycaemic control in critically ill patients. Here we briefly highlight the key function of elevated glucose in critically ill patients, namely, to enable elevation of aerobic glycolysis in rapidly dividing cells. In particular, aerobic glycolysis provides metabolic intermediates necessary for expansion of biomass in immune cells and promotion of tissue repair. Furthermore, we emphasise that insulin may inhibit autophagy, a cell survival process used in the bulk degradation of cellular debris and damaged organelles. These observations provide a rational basis for tolerating elevated glucose levels in certain critically ill patients.

Keywords: Autophagy, Glycaemic control, Hyperglycaemia, Immunometabolism, Insulin, Critical Illness, Sepsis, Glycolysis, Metabolism
\end{abstract}

\section{Introduction}

The hyperglycaemia observed during immune activation was initially seen as an adaptive response linked to the normal stress response. This view was challenged by the findings that insulin therapy in critically ill patients to reduce glucose levels below $110 \mathrm{mg} / \mathrm{dL}(6.1 \mathrm{mmol})$ resulted in a dramatic decrease in mortality [1]. Subsequently, the hyperglycaemia observed during an infection has been described as a defect of glucose homeostasis [2] - a manifestation of pathology that must be treated. A number of observations also support this view. As an example, hyperglycaemia has consistently been associated with an adverse outcome in patients with sepsis, irrespective of their diabetic status [3]. Furthermore, hyperglycaemia is not only associated with activation of inflammatory responses, but in fact has been found to be a causative agent behind the development of certain pathologies. This is particularly

\footnotetext{
* Correspondence: gustav.v.niekerk@gmail.com

Department of Physiological Sciences, Stellenbosch University, Private Bag X1, Matieland, Stellenbosch 7600, South Africa
}

well exemplified by literature on the consequence of hyperglycaemia observed in diabetes. In diabetic patients, continual hyperglycaemia can injure endothelial cells and decrease the capillary bed density in various organs. Mechanistically, hyperglycaemia can increase the formation of advanced glycation end products (AGE) when proteins and lipids become glycated. This may promote a number of detrimental consequences, particularly the binding of AGE to the receptors for advanced glycation end products (RAGE) on immune cells, which may illicit an inflammatory response [4]. There is also evidence that hyperglycaemia might cause mitochondrial damage. In mice, prolonged exposure to an obesogenic diet resulted in insulin resistance, followed by mitochondrial dysfunction associated with an increase in reactive oxygen species production [5]. These changes were also observed when diabetes was chemically induced (treated with streptozotocin), suggesting that increased mitochondrial dysfunction was a result of hyper-glycaemia/lipidaemia [5]. These observations provide a mechanistic foundation by which hyperglycaemia adversely affects critically ill patients, thus providing a rational basis for treating hyperglycaemia in critically ill patients.

However, a number of subsequent studies have failed to show the benefits of glycaemic control [6-11], and some have even found adverse effects associated with strict glycaemic control [12]. The Surviving Sepsis Campaign now recommends targeting blood glucose levels below $180 \mathrm{mg} / \mathrm{dL}$ instead of the previous tight $(<110 \mathrm{mg} / \mathrm{dL})$ glycaemic levels [13]. Hence, the targeting of glucose levels remains a topic that solicits intense debate [14-16]. What is evident, however, is that some patients do seem to benefit from controlled glucose levels, whereas others obviously do not.

A growing understanding of the metabolic needs of activated immune cells provides a new view on the effect of glycaemic control. Here we briefly review both established work as well as recently emerging insights into the metabolism of activated immune cells. It is argued that glycaemic control might in effect exert an 
immune-suppressive effect, which may diminish the host's ability to launch a competent immune response. In fact, the anti-inflammatory effects of insulin might mediate some of the beneficial effects of glycaemic control, suggesting that the metabolism of immune cells may represent novel targets for anti-inflammatory interventions. Secondly, insulin itself may adversely affect patient outcome by inhibiting autophagy, a generic cell survival response. From these considerations, it is clear that glycaemic control may not represent optimum support for all patients.

\section{The metabolic sweet spot}

Lactic acidosis has been ascribed to systemic anaerobic conditions resulting from hypoperfusion manifesting as tissue hypoxia. Indeed, sepsis is often associated with a decrease in blood pressure (in severe cases manifesting as septic shock) which could leave tissue hypoxic. However, evidence emerged that lactic acid build-up poorly correlates with tissue hypoxia. It has been shown that sepsis does not compromise oxygen delivery compared to normal individuals [17]. Indeed, therapies aimed at increasing oxygenation failed to improve clinical parameters $[18,19]$. These observations suggest that lactate buildup is not solely dependent on tissue hypoxia [18, 19]. Other factors may include mitochondrial dysfunction resulting from severe inflammation [20]. In addition, an increase in lactate production by normal cells may also contribute. Indeed, numerous cells make use of 'aerobic glycolysis'-the lactic acid fermentation of sugar despite the abundance of oxygen. Vascular endothelium cells, despite being in direct contact with oxygenated blood, presumably spare the oxygen supply on route for intended tissue [21]. Cancer cells also exhibit an increase in glycolysis despite the presence of oxygen-a phenomena referred to as the 'Warburg effect' (named after Otto Warburg who first described aerobic glycolysis in cancerous cells). Aerobic glycolysis is not unique to proliferating cancer cells, but is also a normal metabolic strategy enacted by various rapidly dividing cells [22], including immune cells [23]. Thus, an increase in aerobic glycolysis conducted by activated immune cells likely contributes to the increase in lactic acid build up through glycolytic activity in other tissues, as well as a decrease in lactic acid clearance.

Yet, anaerobic glycolysis is highly inefficient: whereas oxidative respiration yields 32 moles of ATP for each mole of glucose fully oxidised, glycolysis only produces 2 moles of ATP. This then raises the question why the immune system would make use of such a seemingly ineffective system. One key benefit of making use of aerobic glycolysis relates to energy production: though the efficiency of ATP might be lower for aerobic glycolysis compared to oxidative phosphorylation, the rate at which ATP can be produced is much higher (i.e. more
ATP can be produced by glycolysis than oxidative phosphorylation in a given time unit) [24]. This would suggest that elevated glycolysis in immune cells might represent a metabolic strategy to rapidly increase cellular ATP levels.

Energy production is not the only endpoint of aerobic glycolysis in rapidly dividing cells. Indeed, another function of glycolysis is to provide metabolic intermediates used in other biosynthetic pathways, such as for the synthesis of lipids and nucleotides [22]. This also explains why, in several cancer types, the contribution of glycolysis to ATP production is marginal despite high glucose consumption [25]. The application of aerobic glycolysis is now also understood to play a pivotal role in the activated immune cells of both the innate and adaptive immune systems [26, 27]. As an example, activated monocytes rapidly increase the biosynthesis of fatty acids [28]. Interestingly, following inhibition of fatty acid synthesis with RNA interference, markers of macrophage differentiation were decreased [28], indicating the reliance of differentiation on metabolism. Here, glycolysis can be indispensable in providing the metabolic intermediates (such as acyl-CoA) which can be used for lipid synthesis [22]. The use of glucose for biosynthetic processes is similarly important in cells of the adaptive immune system. As an example, upon activation of a corresponding antigen, B cells rapidly upregulate glucose uptake and glycolysis [29]. Moreover, upregulation of the pentose phosphate pathway (PPP) prior to cells entering the $\mathrm{S}$ phase was also observed. This observation suggests that glucose might be shifted towards biosynthetic pathways, since the PPP is also implemented to provide metabolic intermediates [29].

Taken together, it is clear that glucose plays a central role in the functioning of activated immune cells. Glucose is important for both energy production and maintaining biosynthetic activities associated with the rapid expansion of immune cells and the production of immune modulators/effectors during an infection. This also suggests that hampering glucose supply would likely adversely affect immune cell function.

\section{Addressing the immunological needs: hyperglycaemia}

It is thus pivotal that immune cells receive adequate amounts of glucose. Indeed, energy production by glycolysis can only out-perform oxidative phosphorylation under conditions of high glucose uptake [30]. Similarly, low glucose levels are likely to compromise cellular biosynthetic capacities. In this regard, a number of physiological adaptations exist to augment the glucose supply chain. Firstly, activated immune cells rapidly upregulate the expression of glucose transporters [31], thus enhancing the rate at which glucose is imported. Interestingly, it has also been noted that insulin plays an important role in $\mathrm{T}$ cells, since 
$\mathrm{T}$ cells lacking insulin receptors exhibit a dramatically reduced glycolytic capacity [32]. This is surprising since insulin levels are usually normal or slightly suppressed during sepsis [33]. Regardless, glucose transporters follow Michaelis-Menten kinetics, which implies that substrate concentration (i.e. serum glucose levels) will influence the rate at which glucose is transported into cells.

Serum glucose levels are elevated through a range of physiological mechanisms. Various inflammatory mediators, such as Il-1b and TNF [34], Il-6 [35], as well as type I and II interferons [36], induce insulin resistance. In addition, evidence from mouse models suggests that a decrease in blood flow to muscle might also contribute to the lower glucose consumption in response to a lipopolysaccharide challenge [37]. However, gluconeogenesis in the liver is a major contributing factor towards the development of hyperglycaemia [2]. In fact, an increase in nitrogen secretion reflects the increase in basal metabolic rate (Fig. 1), as the carbon skeleton of amino acids is used to produce glucose, which in turn fuels the elevated metabolic state. Mechanistically, inflammatory cytokines such as Il-6 increase the secretion of glucagon by acting both on the central nervous system as well as directly on islets cells [38]. Taken together, these responses demonstrate the physiological adaptation to the unique metabolic needs of immune cells during an infection, and that altered glucose metabolism during an infection does not represent a 'dysregulation' but a tailored response geared towards the mobilisation of an effective immune response.

These arguments suggest that hyperglycaemia is in service of promoting and sustaining a competent immune response. However, this does not mean that an immune response is necessarily adaptive or desirable. Strict glycaemic control might exert beneficial effects by actually impeding immune function. This might explain why certain patients do benefit from insulin therapy. It is worth noting that the Leuven study was predominantly in patients that were assigned to an ICU after cardiac surgery [1]. In the context of such sterile intervention, attenuating immune function by glycaemic control might be beneficial in avoiding unnecessary inflammation. In contrast, inhibiting immune function in the context of an infection could be disastrous.

\section{Insulin and autophagy}

Insulin, independent of altering glucose levels, may also have unanticipated adverse effects. It has long been noted that an anabolic hormone such as insulin can inhibit the catabolic process of autophagy [39]. In fact, evidence suggests that critically ill patients not only exhibit traits of autophagic insufficiency, but that these effects can be exacerbated by insulin therapy: hepatocytes from critically ill patients receiving insulin as part of glucose control exhibited an $80 \%$ greater reduction in autophagic vacuole formation compared to critically ill patients receiving standard therapy [40]. Such an inhibition of autophagy may have several adverse effects since autophagy plays a pivotal role in both host defence as well as cell survival [41]. In host defence, autophagy plays a key function in the processing of epitopes to be loaded on both MHC I and II, targeting intracellular bacteria for autophagy degradation (referred to as xenophagy). Furthermore, vesicles used for viral replication are also degraded via autophagy. These processes are operative not only in immune cells, but also in non-immune cells, highlighting the potential role of maintaining elevated levels of autophagy during an infection [41].

Autophagy may also play a key role in surviving the adverse conditions faced in a clinical setting, including the removal of damaged or misfolded proteins (aggrephagy) as well as damaged mitochondria (mitophagy) or the clearance of noxious factors such as bacteria and endotoxins by the liver [41]. As an example, critically ill patients demonstrate mitochondrial dysfunction with a concomitant decrease in antioxidant capacity [42]. It is very likely that damaged mitochondria result in an increased formation of radicals, which in turn results in decreased antioxidant capacity. Here, autophagy plays a pivotal role in targeting damaged mitochondria for
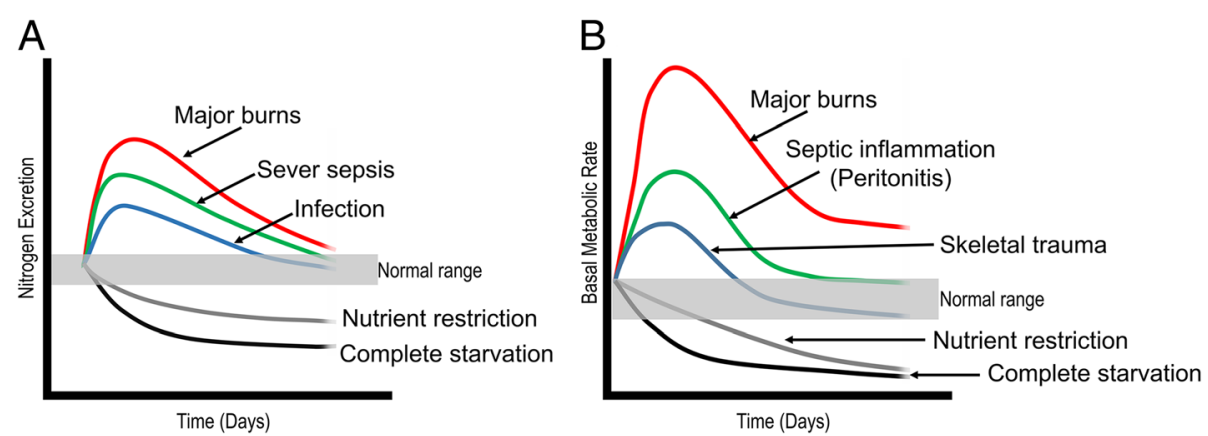

Fig. 1 Activation of an immune response is associated with an increase in metabolic turnover a sustained by increased protein catabolism (b). Redrawn with modification from [48] 
degradation [43]. The key role played by autophagy thus also raises concerns regarding the role of insulin in controlling hypoglycaemia while similarly also inhibiting autophagy. In this study, however, patients were in a fed state, which represents a confounding factor since feeding may suppress autophagy.

\section{Glycaemic control: novel therapeutic strategies}

Activation of an inflammatory response is often accompanied by a decrease in appetite. Yet, despite a decrease in nutrients, rapid expansion of the immune system, as well as the synthesis of immune effectors, must be sustained. In this regard, catabolism in peripheral tissue is directed at maintaining anabolism of the immune system. Here we have argued not merely that hyperglycaemia during immune activation represents an evolutionarily conserved response, but have, in fact, pointed out the reason why elevated glucose levels may in fact play an important function during an infection. However, the manner in which metabolic alteration during an inflammatory insult promotes immune function remains to be formally investigated.

There is also a need to establish safe tolerable levels of glycaemic control as extreme levels of hyperglycaemia can, in fact, have a negative impact on immune function. In a rabbit model of critical illness, maintaining glucoses levels at $13.8-16.6 \mathrm{mmol} / \mathrm{L}$ and $13.875-19.425 \mathrm{mmol} / \mathrm{L}$ adversely affected innate immune cell activity $[44,45]$. Similarly, an increase in glucose levels might be adaptive in the short term, but may exert negative effects (e.g. mitochondrial dysfunction [5]) in a chronic setting. This would suggest that glycaemic control might be beneficial in managing a protracted state. A key question remains whether glycaemic control might represent a strategy for attenuating unwanted immune activation. In this regard, insulin therapy is able to attenuate inflammation dramatically [46]. If such an immune-suppressive effect is indeed mediated by starving immune cells of glucose, it raises the intriguing possibility that novel anti-inflammatory therapies might be developed through targeting of immune cell metabolism. As an example, one meta-analysis [47] has failed to demonstrate any benefit of glycaemic control in patients with sepsis, though a benefit was observed in other ICU settings. This does not, however, indicate that insulin therapy may not have beneficial effects in sepsis. In this regard, insulin therapy may attenuate a life-threatening inflammatory response. There is thus a need to identify individuals who may best benefit from glycaemic control.

Emerging evidence suggests a key role for autophagy within a clinical context. However, more studies are required to investigate the effect of insulin therapy on autophagy. In the study by Vanhorebeek [40] critically ill patients were in a fed state, which would attenuate autophagy, thus representing a confounding factor. Also, if lowering glucose levels prevents cellular damaged caused by elevated glucose levels, it might mitigate the need for higher autophagy. In addition, since autophagy is activated in response to energy stress, it is also possible that hyperglycaemia might attenuate autophagy. Given the potential role of autophagy, establishing the effect of glycaemic control could be of great clinical value. More research is urgently needed to provide a complete picture of the effect exerted by intensive insulin therapy on autophagy.

Acknowledgements

Not applicable.

Funding

None.

Authors' contributions

All authors contributed equally. GVN, TD and AME conceived, drafted and revised the manuscript. All authors read and approved the final manuscript.

Ethics approval and consent to participate

Not applicable.

Consent for publication

Not applicable.

Competing interests

The authors declare that they have no competing interests.

\section{Publisher's Note}

Springer Nature remains neutral with regard to jurisdictional claims in published maps and institutional affiliations.

Published online: 03 August 2017

\section{References}

1. Van den Berghe $G$, Wouters P, Weekers F, Verwaest C, Bruyninckx F, Schetz $M$, Vlasselaers $D$, Ferdinande $P$, Lauwers $P$, Bouillon R. Intensive insulin therapy in critically ill patients. N Engl J Med. 2001;345(19):1359-67.

2. McGuinness OP. Defective glucose homeostasis during infection. Annu Rev Nutr. 2005;25:9-35

3. van Vught LA, Wiewel MA, Klein Klouwenberg PM, Hoogendijk AJ, Scicluna BP, Ong DS, Cremer OL, Horn J, Bonten MM, Schultz MJ, van der Poll T, Molecular Diagnosis and Risk Stratification of Sepsis Consortium. Admission hyperglycemia in critically ill sepsis patients: association with outcome and host response. Crit Care Med. 2016;44(7):1338-46.

4. Goldin A, Beckman JA, Schmidt AM, Creager MA. Advanced glycation end products: sparking the development of diabetic vascular injury. Circulation. 2006:114(6):597-605

5. Bonnard C, Durand A, Peyrol S, Chanseaume E, Chauvin MA, Morio B, Vidal $\mathrm{H}$, Rieusset J. Mitochondrial dysfunction results from oxidative stress in the skeletal muscle of diet-induced insulin-resistant mice. J Clin Invest. 2008; 118(2):789-800

6. Brunkhorst FM, Engel C, Bloos F, Meier-Hellmann A, Ragaller M, Weiler N, Moerer O, Gruendling M, Oppert M, Grond S. Intensive insulin therapy and pentastarch resuscitation in severe sepsis. N Engl J Med. 2008;358(2):125-39.

7. Arabi YM, Dabbagh OC, Tamim HM, Al-Shimemeri AA, Memish ZA, Haddad SH, Syed SJ, Giridhar HR, Rishu AH, Al-Daker MO, Kahoul SH, Britts RJ, Sakkijha $\mathrm{MH}$. Intensive versus conventional insulin therapy: a randomized controlled trial in medical and surgical critically ill patients. Crit Care Med. 2008;36(12):3190-7.

8. De La Rosa GC, Donado JH, Restrepo AH, Quintero AM, González LG, Saldarriaga NE, Bedoya M, Toro JM, Velásquez JB, Valencia JC. Strict glycaemic control in patients hospitalised in a mixed medical and surgical intensive care unit: a randomised clinical trial. Crit Care. 2008;12(5):R120.

9. CollTSS Study Investigators, Annane D, Cariou A, Maxime $V$, Azoulay $E_{\text {, }}$ D'honneur G, Timsit JF, Cohen Y, Wolf M, Fartoukh M, Adrie C, Santre C, 
Bollaert PE, Mathonet A, Amathieu R, Tabah A, Clec'h C, Mayaux J, Lejeune J, Chevret S. Corticosteroid treatment and intensive insulin therapy for septic shock in adults: a randomized controlled trial. JAMA. 2010;303(4):341-8.

10. Preiser J, Devos P, Ruiz-Santana S, Mélot C, Annane D, Groeneveld J, lapichino G, Leverve X, Nitenberg G, Singer P. A prospective randomised multi-centre controlled trial on tight glucose control by intensive insulin therapy in adult intensive care units: the Glucontrol study. Intensive Care Med. 2009;35(10):1738.

11. Kalfon P, Giraudeau B, Ichai C, Guerrini A, Brechot N, Cinotti R, Dequin P, Riu-Poulenc B, Montravers P, Annane D. Tight computerized versus conventional glucose control in the ICU: a randomized controlled trial. Intensive Care Med. 2014;40(2):171-81.

12. Nice-Sugar Study Investigators. Intensive versus conventional glucose control in critically ill patients. N Engl J Med. 2009;2009(360):1283-97.

13. Rhodes A, Evans LE, Alhazzani W, Levy MM, Antonelli M, Ferrer R, Kumar A, Sevransky JE, Sprung CL, Nunnally ME. Surviving Sepsis Campaign: International guidelines for management of sepsis and septic shock: 2016. Intensive Care Med. 2017;43(3):304-77.

14. Marik PE. Tight glycemic control in acutely ill patients: low evidence of benefit, high evidence of harm! Intensive Care Med. 2016;42(9):1475-7.

15. Gunst J, Van den Berghe G. Blood glucose control in the ICU: don't throw out the baby with the bathwater! Intensive Care Med. 2016;42(9):1478-81.

16. Wernly B, Lichtenauer M, Hoppe UC, Jung C. Hyperglycemia in septic patients: an essential stress survival response in all, a robust marker for risk stratification in some, to be messed with in none. J Thorac Dis. 2016;8(7), E621.

17. Ronco JJ, Fenwick JC, Tweeddale MG, Wiggs BR, Phang PT, Cooper DJ, Cunningham KF, Russell JA, Walley KR, Chernow B. Identification of the critical oxygen delivery for anaerobic metabolism in critically III septic and nonseptic humans. JAMA. 1993;270(14):1724-30.

18. Suetrong B, Walley KR. Lactic acidosis in sepsis: it's not all anaerobic: implications for diagnosis and management. CHEST J. 2016;149(1):252-61.

19. Garcia-Alvarez M, Marik P, Bellomo R. Sepsis-associated hyperlactatemia. Crit Care. 2014;18(5):503

20. Ruggieri AJ, Levy RJ, Deutschman CS. Mitochondrial dysfunction and resuscitation in sepsis. Crit Care Clin. 2010;26(3):567-75.

21. Eelen $G$, de Zeeuw P, Simons M, Carmeliet P. Endothelial cell metabolism in normal and diseased vasculature. Circ Res. 2015;116(7):1231-44.

22. Lunt SY, Vander Heiden MG. Aerobic glycolysis: meeting the metabolic requirements of cell proliferation. Annu Rev Cell Dev Biol. 2011;27:441-64.

23. Maciver NJ, Jacobs SR, Wieman HL, Wofford JA, Coloff JL, Rathmell JC. Glucose metabolism in lymphocytes is a regulated process with significant effects on immune cell function and survival. J Leukoc Biol. 2008:84(4):949-57.

24. Pfeiffer T, Schuster S, Bonhoeffer S. Cooperation and competition in the evolution of ATP-producing pathways. Science. 2001;292(5516):504-7.

25. Zu XL, Guppy M. Cancer metabolism: facts, fantasy, and fiction. Biochem Biophys Res Commun. 2004;313(3):459-65.

26. Ganeshan K, Chawla A. Metabolic regulation of immune responses. Annu Rev Immunol. 2014;32:609-34.

27. Loftus RM, Finlay DK. Immunometabolism: Cellular metabolism turns immune regulator. J Biol Chem. 2016;291(1):1-10.

28. Ecker J, Liebisch G, Englmaier M, Grandl M, Robenek H, Schmitz G. Induction of fatty acid synthesis is a key requirement for phagocytic differentiation of human monocytes. Proc Natl Acad Sci U S A. 2010;107(17):7817-22.

29. Doughty CA, Bleiman BF, Wagner DJ, Dufort FJ, Mataraza JM, Roberts MF, Chiles TC. Antigen receptor-mediated changes in glucose metabolism in B lymphocytes: role of phosphatidylinositol 3-kinase signaling in the glycolytic control of growth. Blood. 2006;107(11):4458-65.

30. Vazquez A, Liu J, Zhou Y, Oltvai ZN. Catabolic efficiency of aerobic glycolysis: the Warburg effect revisited. BMC Syst Biol. 2010;4(1):58

31. Calder PC, Dimitriadis G, Newsholme P. Glucose metabolism in lymphoid and inflammatory cells and tissues. Curr Opin Clin Nutr Metab Care. 2007; 10(4):531-40.

32. Fischer HJ, Sie C, Schumann E, Witte AK, Dressel R, van den Brandt J, Reichardt HM. The insulin receptor plays a critical role in T cell function and adaptive immunity. J Immunol. 2017;198(5):1910-20.

33. Marik PE, Raghavan M. Stress-hyperglycemia, insulin and immunomodulation in sepsis. Intensive Care Med. 2004;30(5):748-56.

34. Wen H, Gris D, Lei Y, Jha S, Zhang L, Huang MT, Brickey WJ, Ting JP. Fatty acid-induced NLRP3-ASC inflammasome activation interferes with insulin signaling. Nat Immunol. 2011;12(5):408-15.
35. Kim J, Bachmann RA, Chen J. Interleukin- 6 and insulin resistance. Vitam Horm. 2009:80:613-33.

36. Wada T, Hoshino M, Kimura Y, Ojima M, Nakano T, Koya D, Tsuneki H, Sasaoka T. Both type I and II IFN induce insulin resistance by inducing different isoforms of SOCS expression in 3 T3-L1 adipocytes. Am J Physiol Endocrinol Metab. 2011;300(6):E1112-23.

37. Mulligan KX, Morris RT, Otero YF, Wasserman DH, McGuinness OP. Disassociation of muscle insulin signaling and insulin-stimulated glucose uptake during endotoxemia. PLoS One. 2012;7(1), e30160.

38. Barnes TM, Otero YF, Elliott AD, Locke AD, Malabanan CM, Coldren AG, Brissova M, Piston DW, McGuinness OP. Interleukin-6 amplifies glucagon secretion: coordinated control via the brain and pancreas. Am J Physiol Endocrinol Metab. 2014;307(10):E896-905.

39. Pfeifer $U$. Inhibition by insulin of the physiological autophagic breakdown of cell organelles. Acta Biol Med Ger. 1977:36(11-12):1691-4.

40. Vanhorebeek I, Gunst J, Derde S, Derese I, Boussemaere M, Güiza F, Martinet W, Timmermans J, D'hoore A, Wouters PJ. Insufficient activation of autophagy allows cellular damage to accumulate in critically ill patients. J Clin Endocrinol Metab. 2011;96(4):E633-45.

41. van Niekerk G, Loos B, Nell T, Engelbrecht A. Autophagy-a free meal in sickness-associated anorexia. Autophagy. 2016;12(4):727-34.

42. Brealey D, Brand M, Hargreaves I, Heales S, Land J, Smolenski R, Davies NA Cooper CE, Singer M. Association between mitochondrial dysfunction and severity and outcome of septic shock. Lancet. 2002;360(9328):219-23.

43. Zhang J. Autophagy and mitophagy in cellular damage control. Redox Biol. 2013;1(1):19-23.

44. Weekers F, Giulietti A, Michalaki M, Coopmans W, Van Herck E, Mathieu C, Van den Berghe G. Metabolic, endocrine, and immune effects of stress hyperglycemia in a rabbit model of prolonged critical illness. Endocrinology. 2003;144(12):5329-38.

45. Ellger B, Debaveye Y, Vanhorebeek I, Langouche L, Giulietti A, Van Etten E, Herijgers P, Mathieu C, Van den Berghe G. Survival benefits of intensive insulin therapy in critical illness: impact of maintaining normoglycemia versus glycemia-independent actions of insulin. Diabetes. 2006:55(4):1096-105.

46. Hansen TK, Thiel S, Wouters PJ, Christiansen JS, Van den Berghe G. Intensive insulin therapy exerts antiinflammatory effects in critically ill patients and counteracts the adverse effect of low mannose-binding lectin levels. J Clin Endocrinol Metab. 2003;88(3):1082-8.

47. Griesdale DE, de Souza RJ, van Dam RM, Heyland DK, Cook DJ, Malhotra A, Dhaliwal R, Henderson WR, Chittock DR, Finfer S, Talmor D. Intensive insulin therapy and mortality among critically ill patients: a meta-analysis including NICE-SUGAR study data. CMAJ. 2009;180(8):821-7.

48. Long CL, Schaffel N, Geiger JW, Schiller WR, Blakemore WS. Metabolic response to injury and illness: estimation of energy and protein needs from indirect calorimetry and nitrogen balance. J Parenter Enteral Nutr. 1979;3(6):452-6. 